\title{
microRNA-21 promotes tumor proliferation and invasion in gastric cancer by targeting PTEN
}

\author{
BAO GUI ZHANG, JIAN FANG LI, BEI QIN YU, ZHENG GANG ZHU, BING YA LIU and MIN YAN
}

\begin{abstract}
Shanghai Key Laboratory of Gastric Neoplasms, Department of Surgery, Shanghai Institute of Digestive Surgery, Ruijin Hospital, Shanghai Jiao Tong University School of Medicine, Shanghai 200025, P.R. China
\end{abstract}

Received October 25, 2011; Accepted December 1, 2011

DOI: $10.3892 /$ or.2012.1645

\begin{abstract}
Gastric cancer is one of the most common carcinomas in China. microRNAs, a type of non-coding RNA, are important specific regulators and are involved in numerous bioprocesses of an organism. microRNA-21 (miR-21) has been identified as the most suitable choice for further investigation because it is overexpressed in nearly all solid tumors; furthermore, it has been demonstrated that miR-21 is involved in the genesis and progression of human cancer. It has been reported that PTEN, an important tumour suppressor, is regulated by multiple miRNAs. Thus, in this study we focused on the expression and significance of miR-21 in gastric cancer tissues, and the role of miR-21 in the biological behaviour and the expression of PTEN in gastric cancer cells. Real-time PCR was used to detect miR-21 expression in gastric cancer tissues, the adjacent normal tissues, and the gastric cell lines. The gastric cancer cell line BGC-823 was transfected with pre-miR-21/miR-21 inhibitor to overexpress/downregulate miR-21. The influence of miR-21 on the biological behaviour of gastric cancer cells was evaluated using the CCK-8 kit, FCMs, the scratch healing assay and the transwell test. Western blotting and the Luciferase Reporter Assay were used to evaluate the change of PTEN expression after lowered expression of miR-21 in gastric cancer cell lines. Real-time PCR analysis indicated that miR-21 exhibited higher expression in gastric cancer tissues compared to the adjacent non-tumor tissues. miR-21 expression was significantly associated with the degree of differentiation of the tumour tissues $(\mathrm{P}=0.004)$, as well as local invasion and lymph node metastasis $(\mathrm{P}<0.01)$. After transfection, pre-miR21 BGC-823 cells grew faster than the negative and control groups $(\mathrm{P}<0.01)$. The reduction in
\end{abstract}

Correspondence to: Dr Min Yan or Bing Ya Liu, Shanghai Key Laboratory of Gastric Neoplasms, Department of Surgery, Shanghai Institute of Digestive Surgery, Ruijin Hospital, Shanghai Jiao Tong University School of Medicine, Shanghai 200025, P.R. China

E-mail: ymrjym@yahoo.com.cn

E-mail: byliu@sjtu.edu.cn

Key words: gastric cancer, miR-21, biological behavior, PTEN
miR-21 expression demonstrated a remarkable effect on the biological behaviour of gastric cancer cells $(\mathrm{P}<0.05)$; the premiR-21-transfected cells healed more quickly compared to the control cells in the scratch healing assay, whereas the transwell test indicated that cell migration in vitro was notably inhibited with the downregulation of miR-21 $(\mathrm{P}<0.05)$. The western blot results and Luciferase Reporter Assay demonstrated that PTEN expression was remarkably increased after miR-21 inhibition $(\mathrm{P}<0.05)$. microRNA-21 expression was upregulated in gastric carcinoma tissues and was significantly associated with the degree of differentiation of tumour tissues, local invasion and lymph node metastasis. Overexpression of miR-21 promoted BGC-823 cell growth, invasion and cell migration in vitro, whereas downregulation of miR-21 exhibited a stronger inhibitory effect on the biological behaviour of gastric cancer cells; additionally, miR-21 inhibition may upregulate the PTEN expression level, which indicates that PTEN may be a target gene for gastric cancer initiation and development.

\section{Introduction}

microRNA-21 negatively regulates several targets, and thus impacts tumorigenesis. However, the exact mechanism of action in human gastric carcinoma is poorly understood, and there is currently no direct evidence that demonstrates a correlation between microRNA-21 function and phenotype. In this study, we investigate the function of microRNA-21 as a potent oncomir and probe the relationship between microRNA-21, the targets of microRNA-21, and the phenotypic alterations. microRNAs (miRNAs) are small, non-coding RNAs of 18- to 23-nucleotides found in a diverse array of organisms. They have a broad impact on gene expression through translational repression or post-transcriptional suppression (1). microRNAs play an important role in numerous biological processes, such as development, differentiation, and the cellular stress response (2-4). Recent studies have linked deregulation of miRNAs to various diseases including cancer $(5,6)$. Gastric cancer is the most common malignancy in China and the fourth most common cancer world-wide; moreover, the prognosis for gastric cancer is quite poor. Globally, it is the second and fourth leading cause of cancer-related death in men and women, respectively (7). Despite progress in the development of new management strategies, gastric cancer remains difficult to diagnose at an early stage. According to the International 
Union Against Cancer (UICC) and American Joint Committee on Cancer (AJCC) staging systems (8), nearly $65 \%$ of patients in the USA are initially diagnosed with gastric cancer at an advanced stage (T3/T4), and $85 \%$ of patients have lymph node metastasis (9). The mean survival time for these patients is 24 months and the 5 -year survival rate is only $20-40 \%$ after surgery (10). Thus, the discovery of new biomarkers is of critical importance to allow for early diagnosis of gastric cancer.

Reports have indicated that dysregulation of miRNAs is associated with the formation and progression of gastric cancer $(11,12)$. Therefore, miRNAs are potentially useful biomarkers for clinical diagnosis. Dramatic upregulation of microRNA-21 (miR-21) has been reported in numerous types of cancer (13-15). Therefore, miR-21 is recognized as an oncomir. Several targets of miR-21 have been experimentally validated, including PDCD4 (16) and RECK (17); however, ectopic expression of these targets may exert differing functional effects on tumorigenesis. Moreover, uncontrolled proliferation, lack of apoptosis, and invasiveness, which are all modulated by miR-21, have also been identified in tumorigenesis, leaving several questions unanswered. For example, PTEN is reported to be a direct target of miR-21 in HCC, but the phenotype alteration caused by miR-21-mediated PTEN regulation remains unclear. In this study, the mechanism of oncomir miR-21 in gastric cancer was studied with respect to the regulation of PTEN. We discovered that miR-21 expression was markedly increased in gastric cancer tissues compared to normal tissues. More importantly, we demonstrated that miR-21 promoted gastric cancer cell proliferation by directly targeting PTEN, thereby increasing gastric cancer cell invasiveness by directly targeting PTEN.

\section{Materials and methods}

Cell lines, cell culture, and human tissue samples. The human gastric cancer cell lines SGC-7901, MKN-28, MKN-45, and AGS were purchased from Shanghai Institutes for Biological Sciences, Chinese Academy of Sciences, and NCI-N87, BGC-823, HTB-103, CRL-5974, and CRL-5971 were purchased from the American Type Culture Collection (ATCC). The immortalized normal gastric mucosal epithelial cell line GES-1 was a gift from Professor Feng Bin (Sichuan University, Chengdu, China). The cells were routinely cultured in RPMI-1640 supplemented with $10 \%$ heat-inactivated fetal bovine serum (FBS), $100 \mathrm{U} / \mathrm{ml}$ penicillin and $100 \mu \mathrm{g} / \mathrm{ml}$ streptomycin in a humidified cell incubator with an atmosphere of $5 \% \mathrm{CO}_{2}$ at $37^{\circ} \mathrm{C}$. Cells growing at an exponential rate were used for the experiments.

Fresh frozen human tumor samples and human nonneoplastic gastric tissues were obtained from 30 patients with gastric cancer undergoing radical gastrectomy at the Department of Surgery, Ruijin Hospital Shanghai Jiao Tong University School of Medicine from May 2005 to August 2008. None of the patients had received preoperative treatment, such as radiation therapy or chemotherapy. Sections from each specimen were independently examined by two pathologists, and histological typing was performed using Lauren's classification. TNM classification of malignant tumors was assigned in accordance to the International Union Against Cancer (1997).
RNA isolation and miRNA cloning. Total RNA was extracted and isolated from tissue samples or cell lines using either the mirVana miRNA isolation kit (Ambion, Austin, TX) or the TRIzol method. The quality and quantity of the RNA samples were assessed by standard electrophoresis and spectrophotometric methods. The expression level of mature miR-21 was measured by qRT-PCR according to the TaqMan ${ }^{\circledR}$ MicroRNA Assay protocol (Applied Biosystems) and normalized using U6 small nuclear RNA (RNU6B; Applied Biosystems) by the $2^{-\Delta \mathrm{Ct}}$ method. The relative expression ratio of miR-21 in each paired tumour to non-tumour tissue sample was calculated using the $2^{-\Delta \Delta C t}$ method. The miR-21 expression level was defined as being up-regulated in tumour tissue with a relative expression ratio $>1$, and was defined as downregulated in tumour tissue with a relative expression ratio $<1$.

Transfection with antisense oligonucleotides. The stabilityenhanced miRNA precursor that mimicks miR-21 and the control non-specific miRNA precursor (pre-miR precursor, negative control) and the miR-21 inhibitor (miR-21 inhibitor, negative control) were purchased from Shanghai GenePharma Co., Ltd. BGC-823 cells were trypsinised, counted, and seeded onto 6-well plates the day prior to transfection to ensure $50 \%$ cell confluence on the day of transfection. Transfection of miRNA precursors/inhibitors into BGC-823 cells was performed using Lipofectamine 2000 (Invitrogen) in accordance with the manufacturer's advised procedure. The miRNA precursors/inhibitors were used at a final concentration of $100 \mathrm{nM}$. At $48 \mathrm{~h}$ post-transfection, qRT-PCR and western blot analysis were performed. Transfection efficiency was monitored by the transfection of Cy3-labeled pre- $\mathrm{miR}^{\mathrm{TM}}$ negative control \#1 (Ambion).

Cell proliferation assay. Cell proliferation was monitored by the colorimetric water-soluble tetrazolium salt (CCK8) assay using a Cell Counting Kit-8 (Dojindo) according to the manufacturer's instructions. At $24 \mathrm{~h}$ post-transfection with miR-21 pre-cursor/inhibitor or control oligotides, BGC-823 cells were seeded onto 96 -well plates $\left(2 \times 10^{3}\right.$ cells/well), and cell proliferation was documented every $24 \mathrm{~h}$ for 4 days. The number of viable cells was assessed by measurement of the absorbance at $450 \mathrm{~nm}$ using a Safire ${ }^{2}$ microplate reader (TECAN).

Cell cycle and apoptosis analysis. At $48 \mathrm{~h}$ post-transfection with the miR-21 precursor/inhibitor or control precursor $(100 \mathrm{nM})$, BGC-823 cells were collected by trypsinisation and washed with phosphate-buffered saline (PBS). For cell cycle analysis, the cells were fixed with $75 \%$ ethanol and stored at $4^{\circ} \mathrm{C}$ overnight. The following day, fixed cells were washed with PBS, treated with RNase A (50 $\mu \mathrm{g} / \mathrm{ml})$, and stained with propidium iodide (PI) $(50 \mu \mathrm{g} / \mathrm{ml})$ for $30 \mathrm{~min}$ in the dark. The stained cells were analyzed by flow cytometry (FACSCalibur, BectonDickinson). The cell debris and fixation artifacts were gated out and the cell populations at the $\mathrm{G} 0 / \mathrm{G} 1, \mathrm{~S}$, and $\mathrm{G} 2 / \mathrm{M}$ phases were quantified using the Flowjo7.6.2 (Treestar). At least 10,000 cells in each sample were analyzed to obtain a measurable signal. For apoptosis analysis, an Annexin-V-FITC Apoptosis Detection Kit I (BD Pharmingen) was used according to the manufacturer's instructions. In brief, cells were washed with PBS and resuspended in $1 \mathrm{X}$ binding buffer at a concentration 
of $1 \times 10^{6}$ cells/ml; next, $5 \mu 1$ of FITC Annexin-V and $5 \mu 1$ PI were added to $100 \mu \mathrm{l}$ of the cell suspension and the samples were incubated for $15 \mathrm{~min}$ in the dark, after incubation, $400 \mu \mathrm{l}$ $1 \mathrm{X}$ binding buffer was added. Apoptosis was analyzed by flow cytometry (FACSCalibur, Becton-Dickinson) using the CellQuest software (Becton-Dickinson). The cells undergoing apoptosis were Annexin-V-FITC-positive and PI-negative.

Cell migration assay. Migration of BGC-823 cells was assessed using the QCM ${ }^{\mathrm{TM}}$ 24-Well Colorimetric Cell Migration Assay Kit (Millipore) according to the manufacturer's instructions. Briefly, at $24 \mathrm{~h}$ post-transfection with miR-21 inhibitor or control $(100 \mathrm{nM}), 2 \times 10^{4} \mathrm{BGC}-823$ cells in $300 \mu \mathrm{l}$ serumfree medium were added to the upper chamber. A volume of $0.5 \mathrm{ml}$ of $10 \%$ FBS-containing medium was then added to the lower chamber as a chemoattractant. Cells were incubated for another $36 \mathrm{~h}$ at $37^{\circ} \mathrm{C}$, and non-migrating cells on the upper surface of the membrane were then scraped off with cotton swabs. Cells that migrated to the bottom of the membrane were stained for $30 \mathrm{~min}$ with the cell stain provided in the assay kit. Stained cells were visualised under a microscope. To minimize the bias, at least three randomly selected fields were quantified using x100 magnification, and the average number of cells was taken. For scratch wound-healing motility assays, BGC-823 cells were seeded on 6-well plates and allowed to grow to confluence. Confluent monolayers were scratched with a pipette tip and maintained under standard conditions for 24-48 h. Plates were washed once with fresh medium to remove non-adherent cells, and then photographed. Cells were treated with precursor $(100 \mathrm{nM})$ for $24 \mathrm{~h}$ before wounding as well as throughout the assay period.

Cloning of 3'UTR of PTEN into pMIR-REPORT luciferase vector. Total-RNA from BGC-823 cells was initially reverse transcribed into cDNA with Oligo $(\mathrm{dT})_{16}$, which was used as the template, and wild-type and mutant PTEN 3'-UTRs were amplified by 5'-GGCACTAGTTATACTGGT TCACATCCTACCCCTTTGCACTTGTGGCAACAGAT AAGTTTGCAGTTGGCTAAGAGAGGTT-3', and 5'-GAT AAGCTTCATTCCCCTAACCCGAATACATGCATTAG AATGTAGCAAAACCCTTCGGAAACCTCTCTTAGCC AACTGC-3'; 5'-GGCACTAGTTATACTGGTTCAC ATCCTACCCCTTTGCACTTGTGGCAACAGCTGAAT CTGCAGTTGGCTAAGAGAGGTT-3', and 5'-GATAAG CTTCATTCCCCTAACCCGAATACATGCATTAGAAT GTAGCAAAACCCTTCGGAAACCTCTCTTAGCCAAC TGC-3'. The final pieces of wild-type and mutant PTEN 3'-UTRs were cloned into the SpeI and HindIII sites of the pMIR-REPORT luciferase vector (Ambion) and named $\mathrm{pMIR} / \mathrm{PTEN}$ and $\mathrm{pMIR/PTEN/mut,} \mathrm{respectively.} \mathrm{Both}$ constructs were verified by sequencing.

Luciferase activity assay. BGC-823 cells were cultured in 6 -well plates, and each was transfected with $1 \mu \mathrm{g}$ of either $\mathrm{pMIR/PTEN} \mathrm{vector} \mathrm{or} \mathrm{pMIR/PTEN/mut} \mathrm{vector} \mathrm{containing}$ Firefly luciferase along with $0.05 \mu \mathrm{g}$ of the pRL-TK vector (Promega) containing Renilla luciferase and $30 \mathrm{nM}$ miR-21 inhibitor or control oligonucleotide. Transfection was performed using Lipofectamine 2000 (Invitrogen). At $24 \mathrm{~h}$ post-transfection, relative luciferase activity was calculated by normalising the Firefly luminescence to the Renilla luminescence using the Dual-Luciferase Reporter Assay (Promega) according to the manufacturer's instructions.

Western blot analysis and immunohistochemistry. Cultured cells were lysed using RIPA buffer (Pierce) in the presence of Protease Inhibitor Cocktail (Pierce). Tissue samples were lysed using the T-PER Tissue Protein Extraction Reagent (Pierce) in the presence of Protease Inhibitor Cocktail (Pierce). The protein concentration of the lysates was measured using a BCA Protein Assay Kit (Pierce). Equivalent amounts of protein were resolved and mixed with $5 \mathrm{X}$ Lane Marker Reducing Sample Buffer (Pierce), electrophoresed in a $12.5 \%$ SDS-acrylamide gel, and transferred to Immobilon-P Transfer Membrane (Millipore). The membranes were blocked with $5 \%$ non-fat milk in Tris-buffered saline and then incubated with mouse anti-human PTEN monoclonal antibody (Abcam) followed by horseradish peroxidase-conjugated secondary antibody (Abcam). Signals were detected with Immobilon Western chemiluminescent HRP Substrate (Millipore). GAPDH (Abcam) served as the loading control.

Tissues were fixed in $10 \%$ neutralised formalin and embedded in paraffin blocks. Sections $(4 \mu \mathrm{m})$ were then prepared for immunohistochemical examination. After deparaffinisation and rehydration, antigen retrieval was performed by boiling in $10 \mathrm{mmol} / \mathrm{l}$ of citrate buffer $(\mathrm{pH} \mathrm{6.0)}$ for $10 \mathrm{~min}$. After inhibition of endogenous peroxidase activity for $30 \mathrm{~min}$ with methanol containing $0.3 \% \mathrm{H}_{2} \mathrm{O}_{2}$, the sections were blocked with $2 \%$ bovine serum albumin in PBS for 30 min and incubated with mouse anti-human PTEN monoclonal antibody (Abcam, dilution 1:500). The immune complex was visualised with the Dako REAL ${ }^{\mathrm{TM}}$ EnVision $^{\mathrm{TM}}$ Detection System, Perox-idase/DAB, Rabbit/Mouse (Dako), according to the manufacturer's procedure. The cytoplasm was counterstained with hematoxylin.

Statistical analysis. The relationships between the miR-21 expression level and clinicopathological parameters were analysed using the Pearson $\chi^{2}$ test. For comparisons between two different groups, statistical significance was determined using the Student's t-test. All statistical analyses were performed using the SAS 6.12 software package. A two-tailed value of $\mathrm{P}<0.05$ was considered statistically significant.

\section{Results}

The expression of miR-21 is upregulated in gastric cancer and correlates with clinicopathological parameters. To explore the role of miRNAs in gastric cancer, the expression level of miRNAs was a primary consideration. We used miRNA microarray to analyse the miRNA expression profile of nine gastric cancer cell lines and six normal gastric mucosa, and we identified 146 under-expressed and 17 over-expressed miRNAs in all of the gastric cancer cell lines. It has been previously reported that some dysregulated miRNAs, such as miR-106a, miR-141, miR-143, miR-145, miR-218, miR-31, Let-7a, miR-17-5p, miR-221, miR-93, and miR-136 are altered in gastric cancer (18-24), which is in concordance with our results. Interestingly, we found significant upregulation of miR-21 in gastric cancer cell lines, which has not been previ- 

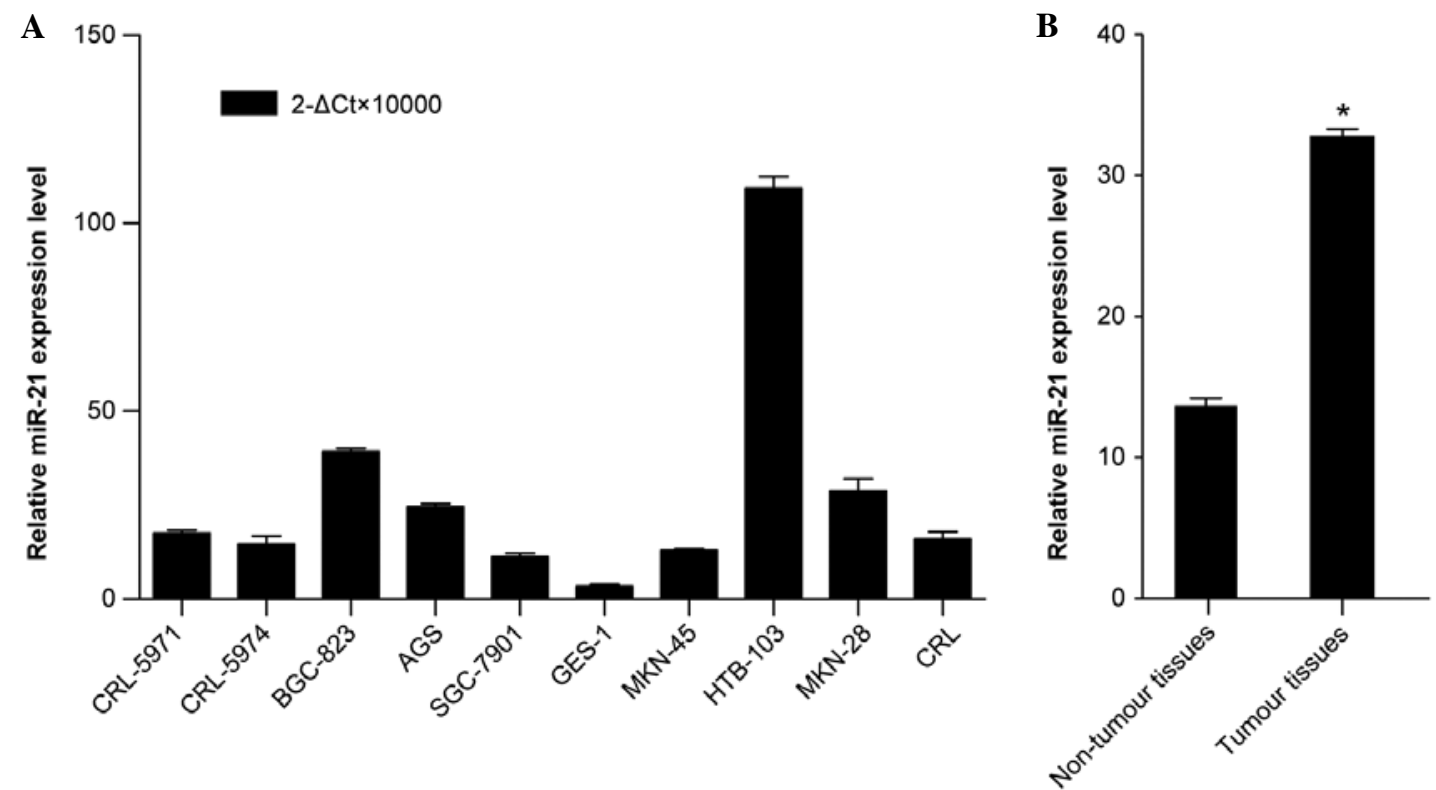

Figure 1. Upregulation of miR-21 expression in gastric cancer tissues and gastric cancer cell lines compared with the corresponding controls. (A) qRT-PCR for miR-21 was performed using nine gastric cancer cell lines and one immortalised normal gastric mucosal epithelial cell line (GES-1). The mean and standard deviation of miR-21 expression levels relative to the miR-21 expression level of GES-1 are shown. The data represent triplicate measurements from single RNA samples (P<0.05, compared with GES-1). (B) qRT-PCR for miR-21 was performed using 30 surgical specimens of gastric cancer tissues and matched with non-tumour tissues. The mean and standard deviation of miR-21 expression levels are shown. The data represent triplicate measurements from single RNA samples $(\mathrm{P}<0.05)$.
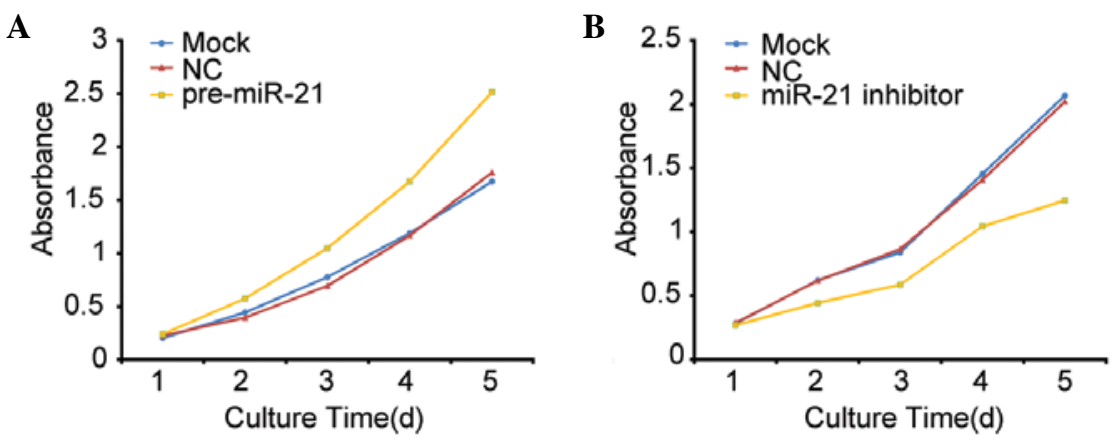

Figure 2. The effect of miR-21 on the proliferation of BGC- 823 cells. Cell proliferation was measured by the CCK 8 assay. BGC- 823 cells were transfected with miR-21 precursor/inhibitor or control at a final concentration of $100 \mathrm{nM}$ and, at $24 \mathrm{~h}$ post-transfection, the CCK8 assay was performed every $24 \mathrm{~h}$ for 4 days. Results are the mean of three independent experiments $\pm \mathrm{SD}(\mathrm{P}<0.05)$. The Cy3-labeled pre-miR ${ }^{\mathrm{TM}}$ negative control \#1 was transfected into BGC-823 cells, and the transfection efficiency was assessed by fluorescence microscope (nuclei were stained with DAPI). Nearly all cells exhibited Cy3 staining, indicating that the miR-21 precursor/inhibitor and control were readily transfected into BGC-823 cells.

ously described. Furthermore, qRT-PCR was performed to detect the miR-21 expression level in the nine gastric cancer cell lines in order to validate the high-expression trend of miR-21 in the gastric cancer cell lines obtained from the miRNA microarray analysis; the results were then compared to one immortalized normal gastric mucosal epithelial cell line (GES-1). As shown in Fig. 1A, miR-21 was significantly upregulated in most gastric cancer cell lines compared with GES-1, thus validating the miRNA microarray results. Similarly, by analyzing the clinical gastric cancer tissues, we found that the average expression level of miR-21 was also significantly upregulated in tumour tissues compared with its matched non-tumour tissues (Fig. 1B). Collectively, these results provided strong evidence that miR-21 is prominently upregulated in gastric cancer. Extensive analysis indicated that among the 30 gastric cancer tissues, $80 \%(24 / 30)$ of the tumour tissues exhibited upregulation of miR-21 compared with the matched non-tumour samples (relative expression ratio $>1.0)$. Furthermore, 55\% (16/30) of the tumour tissues exhibited greater significant upregulation of miR-21 (relative expression ratio $>2.0$ ). Based upon a relative expression ratio of $>2.0$, the miR-21 high-expression group demonstrated a trend toward differentiation. However, miR-21 expression demonstrated no relationship with age, gender, tumour site, or TNM stage (Table I).

Ectopic expression of miR-21 promotes/inhibits the growth of gastric cancer cells. Because miR-21 is markedly upregulated in gastric cancer, it may thus function as a tumour promoter. Therefore, we tested whether over-expression/low-expression of miR-21 in BGC-823 cells affects cell growth. In a CCK8 assay, cells transfected with miR-21 precursor/inhibitor 
Table I. Relationship between miR-21 expression level and clinicopathological parameters.

\begin{tabular}{|c|c|c|c|}
\hline $\begin{array}{l}\text { Clinicopathological } \\
\text { parameters }\end{array}$ & $\mathrm{n}$ & mean $\pm \mathrm{SD}$ & P-value \\
\hline \multicolumn{4}{|l|}{ Age (years) } \\
\hline$\leq 59$ & 14 & $9.00 \pm 9.81$ & 0.675 \\
\hline$>59$ & 16 & $7.60 \pm 8.27$ & \\
\hline \multicolumn{4}{|l|}{ Gender } \\
\hline Male & 17 & $10 . \pm 10.36$ & 0.104 \\
\hline Female & 13 & $5.42 \pm 5.71$ & \\
\hline \multicolumn{4}{|l|}{ Bormann type } \\
\hline I, II & 6 & $7.23 \pm 8.03$ & 0.758 \\
\hline III, IV & 24 & $8.51 \pm 9.23$ & \\
\hline \multicolumn{4}{|l|}{ Location } \\
\hline Middle proximal & 10 & $9.25 \pm 9.76$ & 0.672 \\
\hline Distal & 20 & $7.75 \pm 8.64$ & \\
\hline \multicolumn{4}{|l|}{ Diameter $(\mathrm{cm})$} \\
\hline$\leq 5$ & 18 & $10.58 \pm 10.24$ & 0.138 \\
\hline$>5$ & 12 & $5.53 \pm 5.67$ & \\
\hline \multicolumn{4}{|l|}{ Histological type } \\
\hline Intestinal & 10 & $9.17 \pm 9.25$ & 0.562 \\
\hline Diffuse & 20 & $7.54 \pm 8.32$ & \\
\hline \multicolumn{4}{|l|}{ Depth of invasion } \\
\hline $\mathrm{T} 1, \mathrm{~T} 2$ & 8 & $2.23 \pm 4.16$ & 0.042 \\
\hline $\mathrm{T} 3, \mathrm{~T} 4$ & 22 & $9.8 \pm 10.36$ & \\
\hline \multicolumn{4}{|c|}{ Lymph node metastasis } \\
\hline No & 9 & $3.13 \pm 2.17$ & 0.028 \\
\hline Yes & 21 & $10.3 \pm 9.67$ & \\
\hline \multicolumn{4}{|l|}{ Differentiation } \\
\hline High/middle & 9 & $3.24 \pm 1.47$ & 0.004 \\
\hline Moderate/low & 21 & $10.4 \pm 9.88$ & \\
\hline \multicolumn{4}{|l|}{ TNM stage } \\
\hline I, II & 7 & $5.22 \pm 7.76$ & 0.312 \\
\hline III, IV & 23 & $9.17 \pm 9.16$ & \\
\hline
\end{tabular}

The miR-21 expression level associated with clinicopathological features, including tumour size, lymph node metastasis, local invasion, and tumour-node-metastasis (TNM) stage are shown. Statistical significance was assessed by Pearson $\chi^{2}$ test.

grew more rapidly/slowly than the control group (Fig. 2). The dramatic contrast in proliferative activity indicates that over-expression/low-expression of miR-21 promotes/inhibits the gastric cancer BGC-823 cell growth activity. These results suggest that over-expression/low-expression of miR-21 promotes/inhibits cell growth in vitro. The transfection efficiency was monitored using a Cy3-labeled pre-miR ${ }^{\mathrm{TM}}$ negative control.

Low-expression of miR-21 induces cell cycle arrest in $G 1 / S$ phase and affects gastric cancer cell apoptosis. To elucidate the mechanism of miR-21-mediated cell growth promotion in gastric cancer cells, cell cycle analysis was performed. The results demonstrated that, when compared with the control group, the percentage of miR-21 inhibitor-transfected BGC-823 cells in $\mathrm{G} 1 / \mathrm{S}$ phase increased from $70 \%$ to $91 \%$ $(\mathrm{P}<0.05)$, whereas the percentage of cells in $\mathrm{G} 2 / \mathrm{M}$ phase decreased from 22.9 to $7.6 \%(\mathrm{P}<0.05)$ (Fig. 3A and $\mathrm{B}$ ), and there was a significant difference in the apoptotic rate between the differently treated groups $(12.3$ vs. $6.4 \%, \mathrm{P}<0.05)$ (Fig. 3C and D). These results indicate that low-expression of miR-21 induces G1/S phase arrest in BGC-823 cells, which in turn contributes to the stimulating growth properties of miR-21.

Ectopic expression of miR-21 promotes/inhibits migration of gastric cancer cells in vitro. We further assessed the effects of miR-21 on cell migration, a key determinant of malignant progression and metastasis. As shown in Fig. 4A, miR-21 precursor group scratch wound-healing motility was faster compared to the control group; (Fig. 4B and C) ectopic expression of miR-21 led to significantly decreased migration (miR-21 inhibitor group, $50 \pm 18$ cells per field; control inhibitor group, $100 \pm 28$ cells per field, $\mathrm{P}<0.05)$ of $\mathrm{BGC}-823$ cells. These results propose a functional role for miR-21 in mediating cell migration in gastric cancer and suggest a mechanism by which upregulation of miR-21 potentially contributes to tumour metastasis in gastric cancer.

PTEN is a target of $m i R-21$. Because miR-21 has a pivotal function in gastric cancer, we investigated how this miRNA exerts its role in gastric cancer. We searched for further information regarding its potential target genes that exhibit anti-oncogene properties (13-15); among these genes, PTEN plays a crucial role in the signaling pathways regulating cell adhesion, proliferation, and migration. Therefore, we were able to confirm whether PTEN was also the authentic target gene of miR-21 in gastric cancer. To experimentally validate whether PTEN was a target gene of miR-21 in gastric cancer, a region of the PTEN3'-UTR mRNA was cloned down stream of the Firefly luciferase gene in the pMIR-REPORT luciferase vector. This reporter construct (pMIR/PTEN) was co-transfected into BGC-823 cells with pRL-TK (containing the Renilla luciferase gene to normalize for transfection differences) and miR-21 inhibitor or control. A statistically significant increase of Firefly luciferase activity was observed in BGC-823 cells co-transfected with miR-21 inhibitor and pMIR/PTEN compared with BGC-823 cells co-transfected with control and pMIR/PTEN (Fig. 5A). The resulting construct, $\mathrm{pMIR} / \mathrm{PTEN} / \mathrm{mut}$, was co-transfected into BGC-823 cells with miR-21 inhibitor or control, and the luciferase activity was measured. Importantly, miR-21 could no longer increase Firefly luciferase activity of pMIR/PTEN/ mut (Fig. 5A). These results were confirmed by the following western blot analysis and immunohistochemistry (Fig. 5B-E). Taken together, these results indicate that PTEN-3'-UTR carries the direct binding sites of miR- 21 .

\section{Discussion}

One of the best ways to understand miRNA function is the elucidation of functional targets, and this usually involves analysis of changes in target proteins following either a gain or loss of function of the specific miRNA. Our results indicate 
A

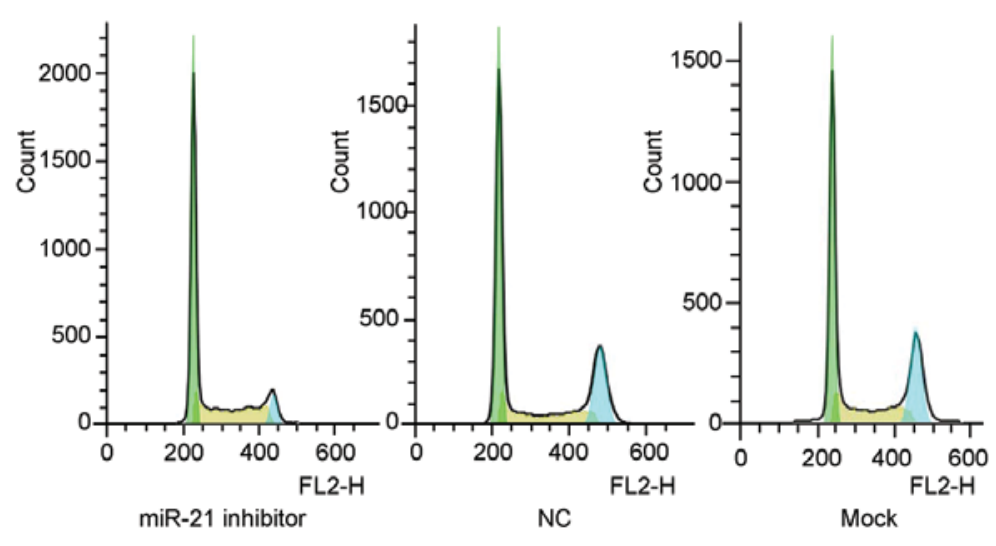

C
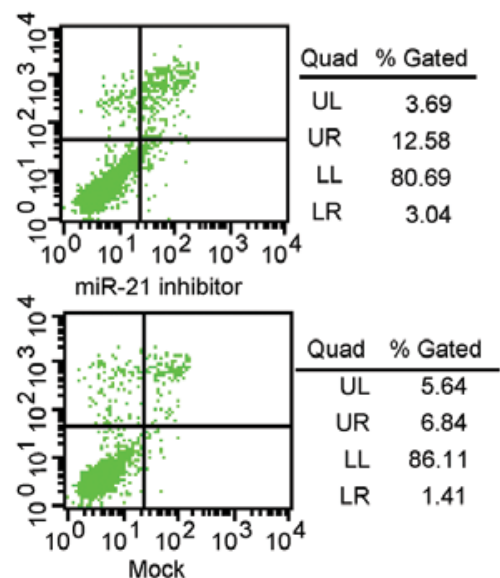

B
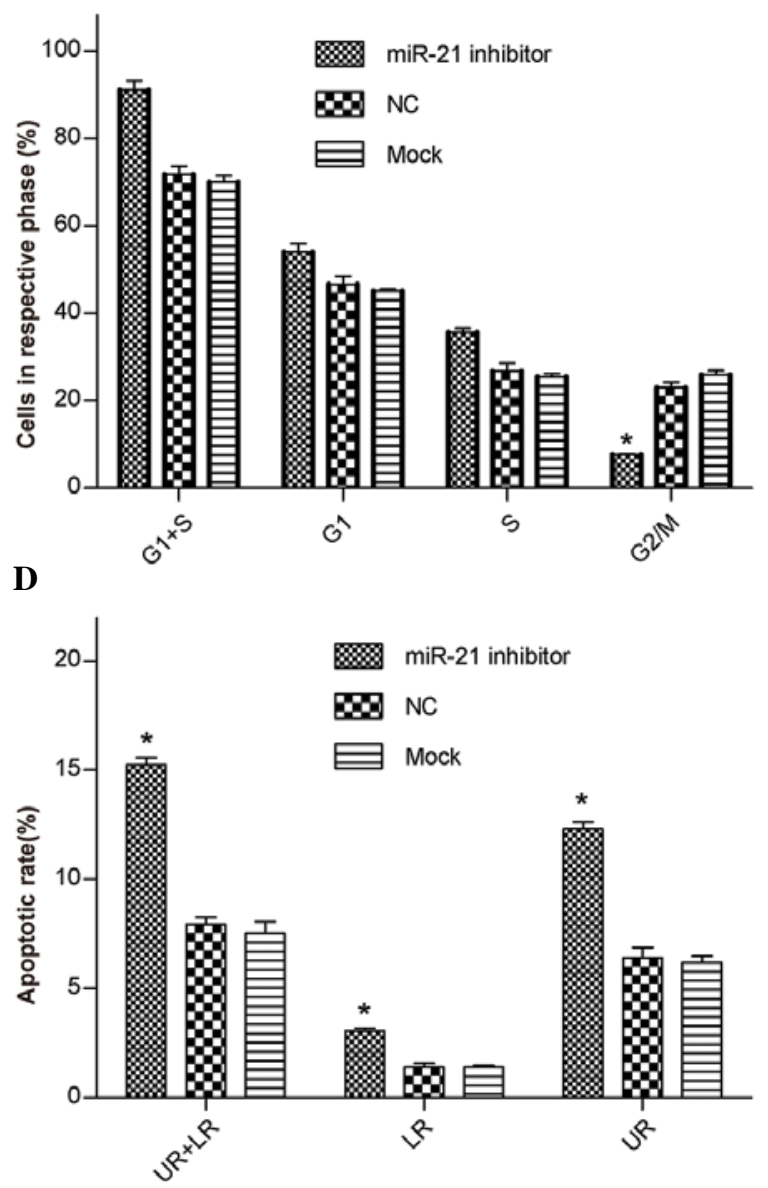

Figure 3. The effect of miR-21 on cell cycle distribution and apoptosis of BGC-823 cells. (A) Proportion of cells in various phases of the cell cycle. (B) Representative histograms depicting cell cycle profiles of BGC-823 cells transiently transfected with miR-21 inhibitor or control (100 nM). Cells were stained with PI and analysed by flow cytometry at $48 \mathrm{~h}$ post-transfection. The results are the mean of three independent experiments $\pm \mathrm{SD}(\mathrm{P}<0.05)$. $(\mathrm{C}) \mathrm{Cells}$ staining positive for Annexin-V-FITC and negative for PI at $48 \mathrm{~h}$ post-transfection were considered to have undergone apoptosis. (D) Representative histograms depicting apoptosis of BGC-823 cells transiently transfected with miR-21 inhibitor or control (100 nM). Average apoptotic rate of three independent experiments $\pm \mathrm{SD}$ are shown

that miR-21 may regulate cell proliferation, apoptosis, and invasiveness by targeting PTEN. These results are somewhat similar to the findings in other types of cancer. However, there is no direct evidence to support a complex correlation among miRNAs, altered cell phenotype resulting from ectopic miRNA treatments, and the many targets of the miRNAs. We found an increase expression of a target gene (PTEN) that resulted from miR-21 inhibition; additionally, we found the inhibition of gastric cancer cell proliferation and the acceleration of apoptosis via anti-miR-21. Thus, we demonstrated that miR-21 generally handles different gastric cancer cell phenotypes by affecting the target, and that one target may contribute to several gastric cancer phenotypes under the control of miR-21. PTEN is a well-known tumor suppressor in multiple cancers, including HCC, and it affects the Akt and ERK signaling pathways (25-27). These pathways are linked to cell survival, proliferation, differentiation, cell migration, and invasion. Hence, this tumor suppressor could either be directly regulated by miR-21 or indirectly through the effect of miR-21 on PTEN. Although PTEN as a target gene of miR-21 has been validated in hepatocellular cancer, breast cancer, and non-small cell lung cancer $(5,28,29)$, Hatley et al $(5)$ confirmed that PTEN was not regulated by niR-21 in non-small cell lung cancer. Additionally, Medina et al (30) demonstrated that through over-expression, miR-21 could lead to pre-B-cell lymphoma, however, Hatley et al (5) suggested that miR-21 high-expression was not sufficient in the non-small cell lung cancer tumorigenesis model. Thus, how do we determine whether miR-21 is tissue specific? We observed an increase of PTEN expression in gastric cancer cells treated with antimiR-21 as compared to untreated cells or cells treated with the scrambled-sequence oligonucleotide. These findings further demonstrate that miR-21, along with its known targets and a few associated genes, forms a complex regulatory network that plays an important role in gastric cancer formation and progression. Therefore, we believe that miR-21 is at the core of a tumorigenic regulatory network at a non-coding RNA level, and may be a new potential target for gastric cancer therapy. Taken together, our findings suggest that the phenotypes of gastric cancer cells (uncontrolled proliferation, increased survival, and invasiveness) are at least partly the result of miR-21 regulation of PTEN. Consequently, suppression of miR-21 may be a novel approach for the treatment of gastric cancer. 
A

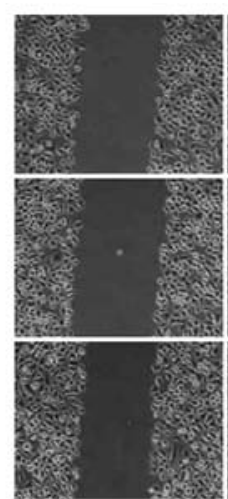

Oh

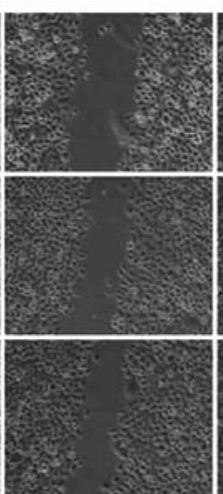

$24 \mathrm{~h}$

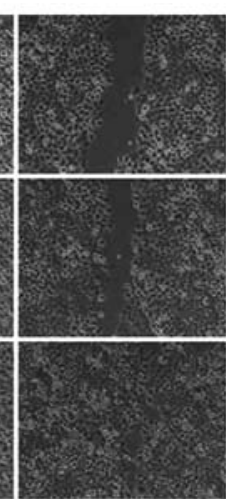

$48 \mathrm{~h}$
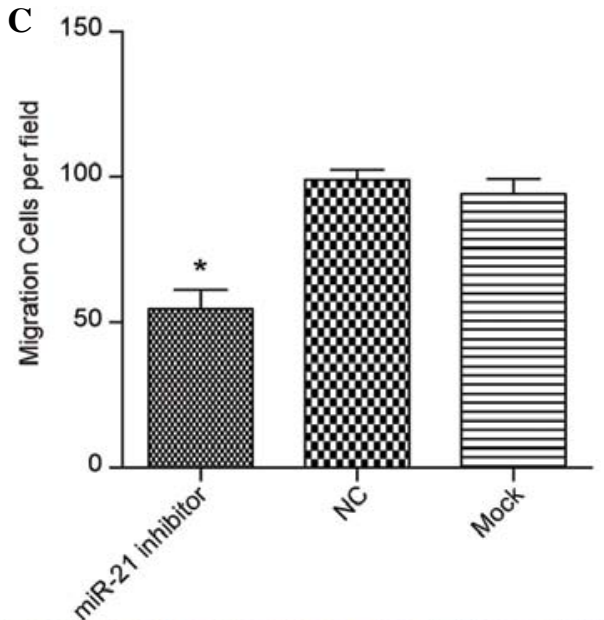

B

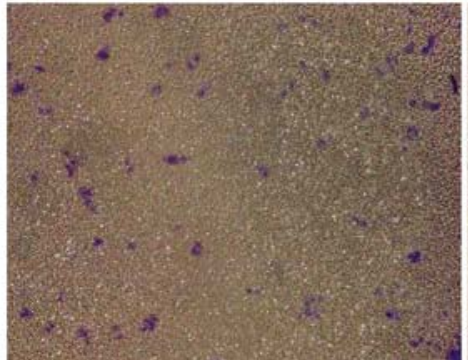

miR-21 inhibitor

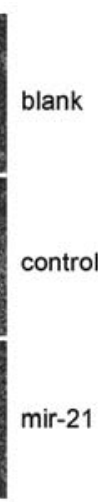

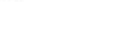

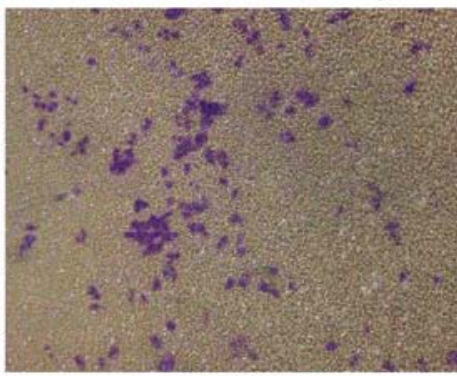

NC

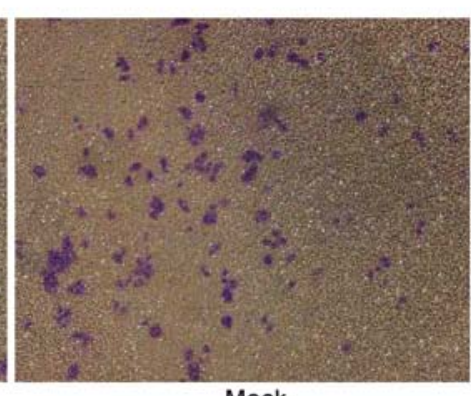

Mock

Figure 4. microRNA-21 promotes migration of BGC-823 cells in vitro. BGC-823 cells were first transfected with miR-21 precursor/inhibitor and control $(100 \mathrm{nM})$ and then subjected to scratch wound-healing motility assays/transwell migration assays. (A) Representative photographs of Scratch wound-healing motility assays. (B and C) Average migratory cell number of three independent experiments $\pm \mathrm{SD}(\mathrm{P}<0.05)$.

A

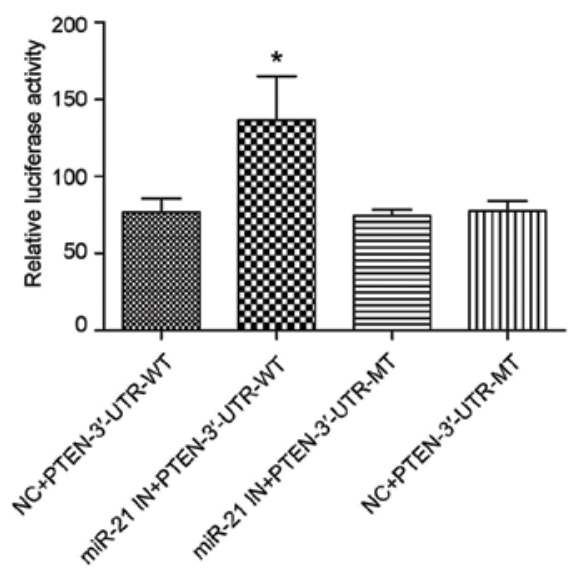

C

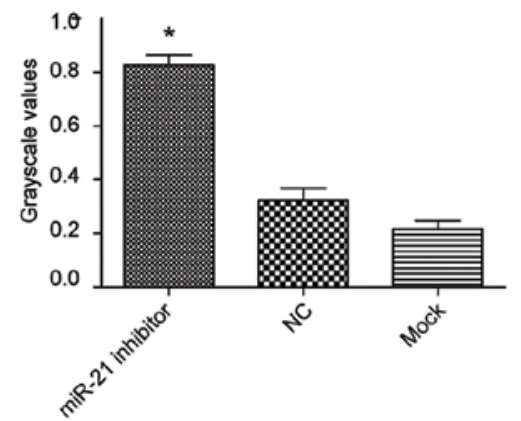

B

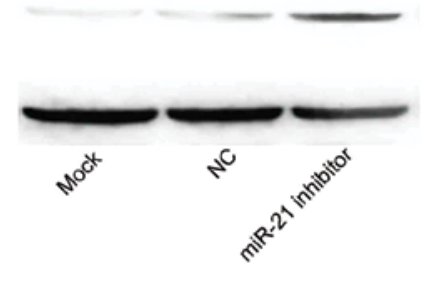

PTEN 53 kDa

GAPDH 36 kDa

D

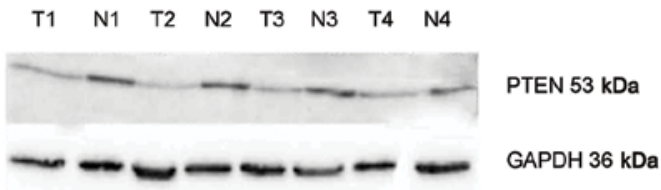

$\mathbf{E}$

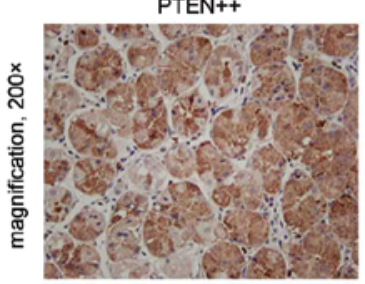

Normal

PTEN+-

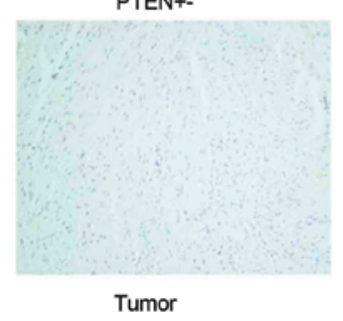

Figure 5. PTEN is a validated target of miR-21. (A) miR-21 inbibitor upregulated luciferase activities controlled by wild-type PTEN-3'-UTR (P<0.05) but did not affect luciferase activity controlled by mutant PTEN-3'-UTR. The results are the mean of three independent experiments \pm SD (P<0.05). (B and C) PTEN protein in BGC-823 cells was detected by western blot analysis at $48 \mathrm{~h}$ post-transfection with miR-21 inhibitor and control (100 $\mathrm{nM}$ ). GAPDH was used as an internal loading control. A reproducible result was obtained in three independent experiments. The results are shown as fold-changes relative to the control inhibitor-transfected BGC-823 cells. (D and E) PTEN expression in gastric cancer tissues and non-tumour tissues determined by western blot analysis and immunohistochemistry. 


\section{Acknowledgements}

This study was supported by grants from the National Natural Science Foundation of China (no. 30872476), the Science and Technology Commission of Shanghai Municipality (no. 10jc1411100,09DZ1950100,09DZ2260200), the Shanghai Key Discipline (S30204) and Key Projects in the National Science and Technology Pillar Program of China (no. 2008BA152B03).

\section{References}

1. Ambros V: The functions of animal microRNAs. Nature 431: 350-355, 2004.

2. Hornstein E, Mansfield JH, Yekta S, et al: The microRNA miR-196 acts upstream of Hoxb8 and Shh in limb development. Nature 438: 671-674, 2005.

3. Sempere LF, Freemantle S, Pitha-Rowe I, et al: Expression profiling of mammalian microRNAs uncovers a subset of brainexpressed microRNAs with possible roles in murine and human neuronal differentiation. Genome Biol 5: R13, 2004.

4. Zhao Y, Samal E and Srivastava D: Serum response factor regulates a muscle-specific microRNA that targets Hand 2 during cardiogenesis. Nature 436: 214-220, 2004.

5. Hatley ME, Patrick DM, Garcia MR, et al: Modulation of K-Rasdependent lung tumorigenesis by MicroRNA-21. Cancer Cell 18: 282-293, 2010.

6. Wang P, Zou F, Zhang X, et al: MicroRNA-21 negatively regulates $\mathrm{Cdc} 25 \mathrm{~A}$ and cell cycle progression in colon cancer cells. Cancer Res 69: 8157-8165, 2009.

7. $\mathrm{Hu} \mathrm{H}, \mathrm{Li} \mathrm{Y,} \mathrm{Gu} \mathrm{J,} \mathrm{et} \mathrm{al:} \mathrm{Antisense} \mathrm{oligonucleotide} \mathrm{against} \mathrm{miR-21}$ inhibits migration and induces apoptosis in leukemic K562 cells. Leuk Lymphoma 51: 694-701, 2010.

8. Crew KD and Neugut AI: Epidemiology of gastric cancer. World J Gastroenterol 12: 354-362, 2010.

9. Dicken BJ, Bigam DL, Cass C, et al: Gastric adenocarcinoma: review and considerations for future directions. Ann Surg 24: 27-39, 2010.

10. Hundahl SA, Phillips JL and Menck HR: The National Cancer Data Base report on poor survival of US gastric carcinoma patients treated with gastrectomy. Cancer 88: 921-932, 2000.

11. Cheung HH, Davis AJ, Lee TL, et al: Methylation of an intronic region regulates miR-199a in testicular tumor malignancy. Oncogene 30: 3404-3415, 2011.

12. Zhang X, Yan Z, Zhang J, et al: Combination of hsa-miR-375 and hsa-miR-142-5p as a predictor for recurrence risk in gastric cancer patients following surgical resection. Ann Oncol 22: 2257-2266, 2011

13. Iorio MV, Ferracin M, Liu CG, et al: MicroRNA gene expression deregulation in human breast cancer. Cancer Res 65: 7065-7070, 2005 .
14. Chan JA, Krichevsky AM and Kosik KS: MicroRNA-21 is an antiapoptotic factor in human glioblastoma cells. Cancer Res 65: 6029-6033, 2005.

15. Si ML, Zhu S, Wu H, et al: miR-21-mediated tumor growth. Oncogene 26: 2799-2803, 2007.

16. Asangani IA, Rasheed SA, Nikolova DA, et al: MicroRNA-21 (miR-21) post-transcriptionally downregulates tumor suppressor Pdcd 4 and stimulates invasion, intravasation and metastasis in colorectal cancer. Oncogene 27: 2128-2136, 2008.

17. Gabriely G, Wurdinger T, Kesari S, et al: MicroRNA 21 promotes glioma invasion by targeting matrix metallopro-teinase regulators. Mol Cell Biol 28: 5369-5380, 2008.

18. Xiao B, Guo J, Miao Y, et al: Detection of miR-106a in gastric carcinoma and its clinical significance. Clin Chim Acta 400: 97-102, 2009.

19. Du Y, Xu Y, Ding L, et al: Down-regulation of miR-141 in gastric cancer and its involvement in cell growth. J Gastroenterol 44: 556-561, 2009.

20. Takagi T, Iio A, Nakagawa Y, et al: Decreased expression of microRNA-143 and -145 in human gastric cancers. Oncology 77: 12-21, 2009.

21. Gao C, Zhang Z, Liu W, et al: Reduced microRNA-218 expression is associated with high nuclear factor kappa B activation in gastric cancer. Cancer 116: 41-49, 2010.

22. Zhang Y, Guo J, Li D, et al: Down-regulation of miR-31 expression in gastric cancer tissues and its clinical significance. Med Oncol 27: 685-689, 2010.

23. Zhang HH, Wang XJ, Li GX, et al: Detection of let-7a microRNA by real-time PCR in gastric carcinoma. World J Gastroenterol 13: 2883-2888, 2007.

24. Petrocca F, Visone R, Onelli MR, et al: E2F1-regulated microRNAs impair TGFbeta-dependent cell-cycle arrest and apoptosis in gastric cancer. Cancer Cell 13: 272-286, 2008.

25. Liu LT, Chang HC, Chiang LC, et al: Histone deacetylase inhibitor up-regulates RECK to inhibit MMP-2 activation and cancer cell invasion. Cancer Res 63: 3069-3072, 2003.

26. Yu J, Zhang SS, Saito K, et al: PTEN regulation by Akt-EGR1ARF-PTEN axis. EMBO J 28: 21-33, 2009.

27. To MD, Perez-Losada J, Mao JH, et al: Crosstalk between Pten and Ras signaling pathways in tumor development. Cell Cycle 4: 1185-1188, 2005.

28. Meng F, Henson R, Wehbe-Janek H, et al: MicroRNA-21 regulates expression of the PTEN tumor suppressor gene in human hepatocellular cancer. Gastroenterology 133: 647-658, 2007.

29. Qi L, Bart J, Tan LP, et al: Expression of miR-21 and its targets (PTEN, PDCD4, TM1) in flat epithelial atypia of the breast in relation to ductal carcinoma in situ and invasive carcinoma. BMC Cancer 9: 163, 2009.

30. Medina PP, Nolde M, Slack FJ, et al: OncomiR addiction in an in vivo model of microRNA-21-induced pre-B-cell lymphoma. Nature 467: 86-90, 2010. 